\title{
Wireless Sensor Network Design for Monitoring and Irrigation Control: User-centric Hardware and Software Development
}

\author{
David Kohanbash ${ }^{1,3}$, George Kantor ${ }^{1}$, Todd Martin ${ }^{2}$, \\ and Lauren Crawford ${ }^{2}$
}

ADDITIONAL INDEX WORDS. nR5, remote, user interface, WSN

SUMMARY. Wireless sensor networks (WSNs) are increasingly becoming a critical tool for growers and researchers. We describe how the technology has advanced, starting with a commercially available WSN node and pushing the technology to make the data more meaningful, actionable and to add advanced irrigation control functionality. User features such as spatial views, custom charts, real-time data access, remote access, irrigation control, alerts, and plant models help create an advanced WSN system that is user centric. Growers and researchers were involved in the design process by directly communicating with the design engineers, and continuously using and testing new features, resulting in a user-centric design and experience. The results of this research are being rolled into a new line of commercial products and is continuously evolving based on user feedback and interaction.

W ireless sensor networks (Fig. 1) are an important tool for monitoring crops and controlling irrigation (Angelopoulos et al., 2011; Bauerle et al., 2013; Coates et al., 2012; Lea-Cox, 2012). A common issue with today's WSN systems is being able to understand the vast amount of collected data. Next generation WSN systems need to help the user understand the data and make the data actionable. One way to make the data actionable is to control irrigation. At the simplest level, users can look at the current sensor data (van Iersel et al., 2013) and use it to decide when to irrigate. Tools that provide real-time data access and custom charts make it easy to identify soil moisture trends, allowing the grower to schedule irrigation events that match crop water needs with greater precision. Advancing remote irrigation control to the next level allows the sensor node that is reading the data in the field to also control irrigation from an onboard solenoid control relay. This allows the grower to remotely apply

\footnotetext{
This paper is part of a series of manuscripts describing the research and development completed by the SCRI-MINDS (Managing Irrigation and Nutrition through Distributed Sensing) project. The authors gratefully acknowledge funding and support from the USDA-NIFA Specialty Crops Research Initiative; Award \#2009-51181-05768.

${ }^{1}$ Robotics Institute, Carnegie Mellon University, 5000 Forbes Avenue, Pittsburgh, PA 15213

${ }^{2}$ Decagon Devices, Inc., 2365 Northeast Hopkins Court, Pullman, WA 99163

${ }^{3}$ Corresponding author. E-mail: dkohanba@cmu.edu.
}

irrigation or modify the irrigation schedule based on the current conditions in the field. To further improve irrigation control, the software system provides "local" set point control, which makes irrigation decisions locally at the node using attached sensors. In this mode, the grower sets the desired volumetric water content and the node will irrigate to that moisture level using feedback from attached soil moisture sensors. Lastly, there are a set of customizable grower tools that allow nodes to irrigate based on externally derived data. This "global control" allows data from other nodes or grower tools to be used for controlling irrigation. Global control brings precision irrigation to an entirely new level by allowing plant physiological grower tool models to run in real time and determine plant water requirements (Kohanbash et al., 2012a).

\section{Irrigation node development}

Two data loggers (also referred to as nodes) that are capable of reading sensors and controlling irrigation solenoids were developed as part of this project (Fig. 2) (Kantor et al., 2012). They are both based on the Em50R data logger (Decagon Devices,
Pullman, WA). This data logger was chosen as it operates reliably in field conditions, has a long battery life (battery life depends on frequency of sensor data collection. Typically 5 AA batteries last a full growing season), and robust long range (typically greater than $4 \mathrm{~km}$ line-of-sight) radio telemetry in outdoor environments. It can support up to five different sensors at a time, and there are many different sensor types that the manufacturer provides that provide multifunctional use for the node. By building on this solid platform, we are able to leverage the existing commercial product to improve the reliability of the new system that allows for irrigation control and remote configuration of node settings.

The two nodes that were developed include the nR5 (Decagon Devices) node, which is capable of controlling $24-\mathrm{V}$ (alternating current) solenoids, and based on user feedback the nR5-DC was developed that can control $12-\mathrm{V}$ (direct current) latching solenoids. Solenoids connected to the nR5-DC are powered by the node, so growers do not need to distribute electrical wires for irrigation solenoids, providing time and money savings for the grower. A new user software interface called Sensorweb has been developed that allows the user to interact with the WSN and make the data actionable.

In the transition from a node that only logs and reports data to an intelligent node that can control irrigation, it was determined that a new underlying application level communications protocol needed to be developed built on the serial radio interface provided by the nodes radio. The decision to develop a new application level protocol for node packets is based on the need to conserve power and for reliability. Standard transport level communication protocols (TCP/IP, 6LoWPAN, etc.) are not suitable for a sensor network-based control system: they do not account for the need to have two-way communications with nodes that are not always awake, and they are bloated with power-consuming features that are

\begin{tabular}{llll}
\hline $\begin{array}{l}\text { Units } \\
\text { To convert U.S. to SI, } \\
\text { multiply by }\end{array}$ & U.S. unit & SI unit & $\begin{array}{l}\text { To convert SI to U.S., } \\
\text { multiply by }\end{array}$ \\
\hline 1.6093 & mile(s) & $\mathrm{km}$ & 0.6214
\end{tabular}


Farm, Nursery or Greenhouse Zone

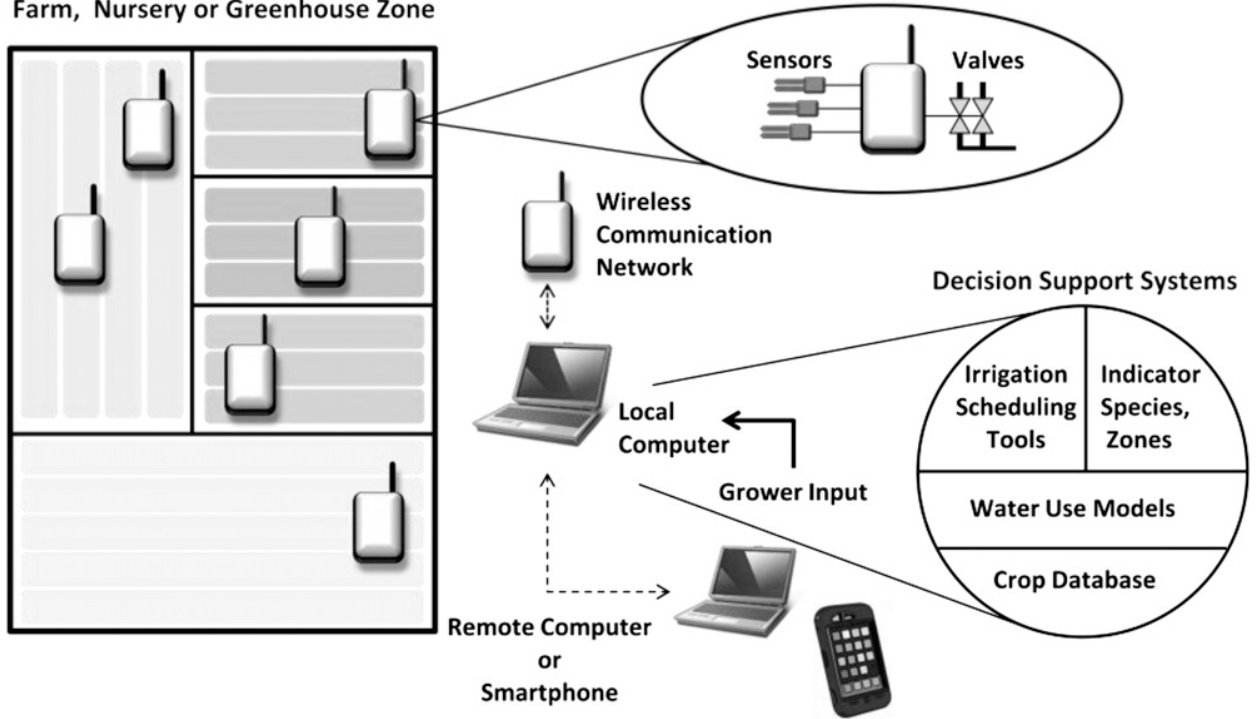

Fig. 1. Image of a basic wireless sensor network showing the remote access (Lea-Cox, 2012). Node supports up to five sensors plus has one onboard relay/solenoid controller that can be used to control multiple solenoids simultaneously.

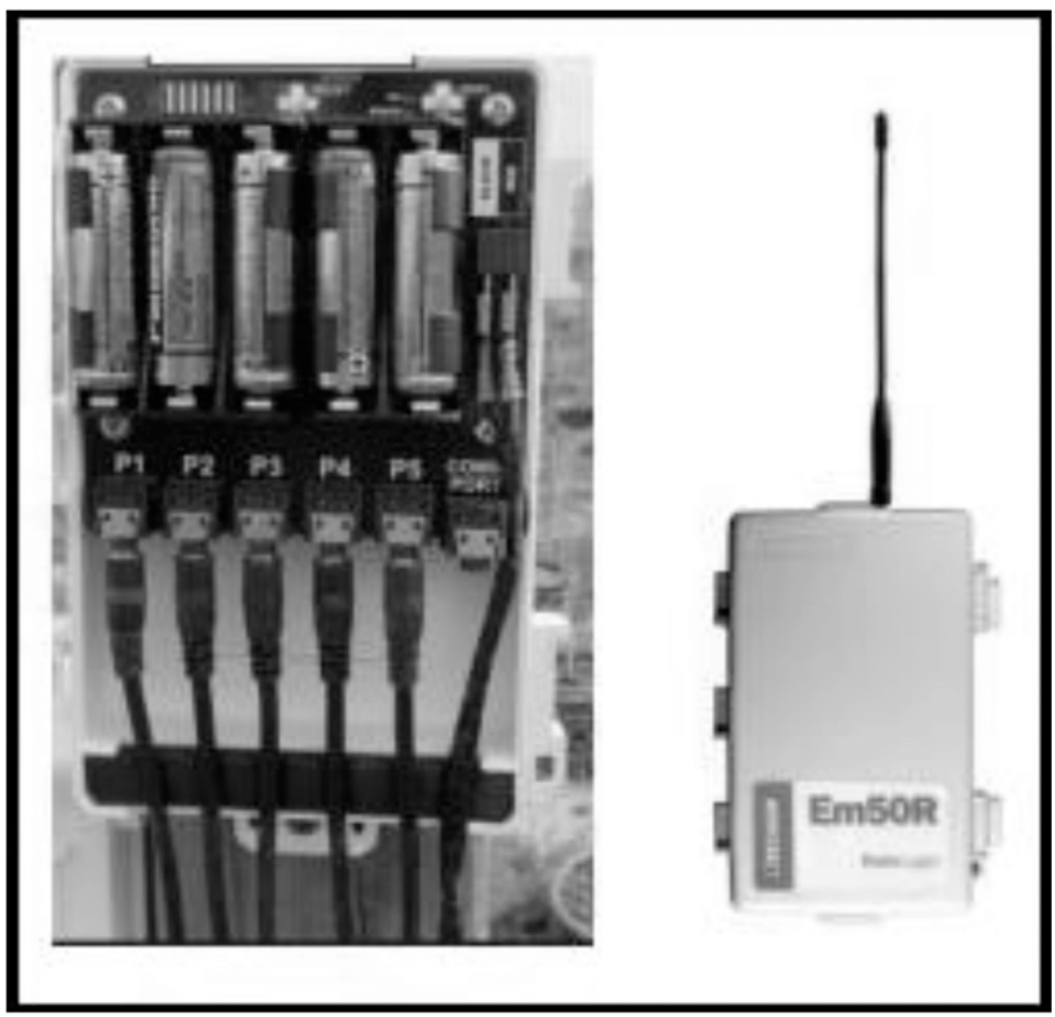

Fig. 2. Decagon Devices (Pullman, WA) nodes. The right image is an Em50R, which the new nodes are based on. The left image is the interior of the new nR5-DC.

not necessary for this system. By developing a custom protocol, we can fine tune performance and battery life. The nodes use a $900-\mathrm{MHz}$ radio (XSC; Digi, Minnetonka, MN) for direct point-to-point communications. To decrease node power usage, the data packets in the new protocol had to be 64 bytes or less so that the complete data packet would not get split into multiple packets for data transmission. We chose to transmit binary data instead of ASCII characters for most of the communication packet contents, which makes the raw packets harder to read as a human but allows for more information to be put into a 64-byte packet. This packing minimizes the number of packets that need to be transmitted to further increase battery life. Each packet 
starts with a common character followed by the packet identification (ID) (Table l), the node serial number and a timestamp. After the header comes the packet specific data followed by the footer. The footer contains a cyclic redundancy control (CRC) value for data integrity, the number of attempts that the node made to transmit the packet, and an end of packet character (Fig. 3). If the data does not require the full field size, it is padded so that the packet size for a given packet type remains the same and can be properly parsed. Every time the node is turned on and then again every $24 \mathrm{~h}$ thereafter, the node publishes its current configuration. This helps make sure that the information in the base station is always up to date and also allows nodes to automatically be detected and added into the base station.

When a packet is sent from the node to the base station, the base station transmits a confirmation packet so the node knows that the packet was received correctly. The confirmation packet always has the attempt number and CRC value for the packet it is confirming. When the base station sends a new configuration packet to the node, the node saves the settings and then sends out a packet with the existing settings that the base station can verify. This is then followed by the base sending a confirmation packet as it does when receiving any packet from a node. After attempting to send a packet 10 times,

Table 1. List of the various packets used to communicate between the nodes and base stations. The identification (ID) field is the letter that is used within the packet to identify what type of packet it is.

\begin{tabular}{|c|c|c|c|}
\hline$\underline{\text { ID }}$ & Packet name & Source ${ }^{\mathrm{z}}$ & Description \\
\hline A & Test & $\mathrm{N}$ & $\begin{array}{l}\text { A test packet that the node can send out } \\
\text { to verify connection to the base }\end{array}$ \\
\hline B & Data & $\mathrm{N}$ & Data packet sent from the node \\
\hline $\mathrm{C}$ & Node configuration & $\mathrm{N} / \mathrm{B}$ & $\begin{array}{l}\text { Contains current general node settings or } \\
\text { can be sent by the base to change the } \\
\text { nodes settings }\end{array}$ \\
\hline $\mathrm{D}$ & Location & $\mathrm{N} / \mathrm{B}$ & $\begin{array}{l}\text { Contains optional node location } \\
\text { information. This can also be sent from } \\
\text { the base to configure the node settings }\end{array}$ \\
\hline $\mathrm{E}$ & Irrigation configuration & $\mathrm{N} / \mathrm{B}$ & $\begin{array}{l}\text { Contains current irrigation settings or } \\
\text { can be sent by the base to change the } \\
\text { nodes settings }\end{array}$ \\
\hline $\mathrm{F}$ & Device status & $\mathrm{N}$ & $\begin{array}{l}\text { Contains node statistics and reports any } \\
\text { errors }\end{array}$ \\
\hline G & Control status & $\mathrm{N}$ & $\begin{array}{l}\text { The node sends this out every time an } \\
\text { irrigation event starts or stops. This } \\
\text { message tells the base how long the } \\
\text { irrigation was and if there was any } \\
\text { errors }\end{array}$ \\
\hline $\mathrm{H}$ & Control override & B & $\begin{array}{l}\text { This message is sent by the base station to } \\
\text { initiate a manual irrigation event }\end{array}$ \\
\hline $\mathrm{X}$ & Base confirmation & B & $\begin{array}{l}\text { The base station sends this packet as } \\
\text { a response to any incoming packet } \\
\text { from a node }\end{array}$ \\
\hline $\mathrm{Y}$ & Node confirmation & $\mathrm{N}$ & $\begin{array}{l}\text { The node uses this packet as an extra } \\
\text { verification step when irrigation events } \\
\text { are scheduled }\end{array}$ \\
\hline
\end{tabular}

${ }^{2} \mathrm{~N}=$ node sends the packet, $\mathrm{B}=$ base station sends the packet, $\mathrm{N} / \mathrm{B}=$ node and base station both send the packet.

the sender will give up. Ten times was chosen as a balance between trying to get packets when there is poor radio communications and minimizing transmissions to conserve battery life. This process is important for two reasons. The first is to insure that data and irrigation settings are correct, if the node irrigates longer than commanded because of a bad packet that can cause problems. The second reason is that agricultural environments often have hills, valleys, trees, building, power lines, and long distances that can lead to imperfect communication between the node and the base station. By transmitting data multiple times, the probability of having successful radio communications increase.

To conserve battery power, the nodes spend most of the time in a low power sleep mode and only awake briefly to transmit data at a user-defined sensor measurement interval. One resulting challenge to having two-way communications between a base station that is typically connected to a computer in an office/shed and a node is that the node needs to be awake to receive commands from the base station. To solve this problem, the confirmation packet from the base station has a field that can tell the node to stay awake for a few seconds. This way when the base station sends a confirmation packet to a node it can request extra time before the node/radio goes to sleep so that it can follow up the confirmation packet with another packet for changing settings on the node. Using this strategy of embedding commands into the confirmation packet can be expanded for any other parameter that must be sent regularly. Another example of this is the base station sends an irrigation enable/disable flag in the confirmation packet that is used for global control irrigation to determine if irrigation is required or not. This lets us put commands in a packet that is already be transmitted instead of using power to send additional packets for that command.

\section{WSN software development}

The software and user interface (Sensorweb) are critical for users to

<start delimiter $><$ packet $|D\rangle<$ Node Serial Number $><$ Packet Time $><$ Payload $><C R C><$ Attempt \# $><$ end delimiter $>$

Fig. 3. Packet structure for radio packets from node to base station. The payload field varies significantly between packet types. 
get the full benefit of any WSN system. This software development also led to the design of an advanced interface that was the result of a great deal of consultations and beta testing by the growers in the project with direct communication to the software engineering team. Features were added to this system (and removed) based on user feedback and by observing user practices. This web-based software allows for remote access over the Internet in a familiar web-based format. This allows growers to monitor and control their irrigation from anywhere where they can get an Internet connection. The interface is designed to work with both standard computers and mobile devices (such as smart phones and tablets). There are four main software components to the WSN base station: the database, the node communication software, the user interface, and the grower tool system.

Although all parts of the system were designed for direct user interaction, the interface is the one piece that the user has the most exposure to. This means that the interface needs to be well designed with intuitive features and easy to customize. One of the complexities in tailoring the user interface to the grower is that every grower is unique. The user interface needs to be flexible enough to cover the needs of as many different growers and situations as possible, from field, to container-nursery to greenhouse environments. It is important that the first thing a user sees provides them with easily to understand and useful information. After a user logs into their farms' passwordprotected website, the first screen that is shown is a spatial view of the farm (Fig. 4) that is color coded based on a user-selected measurement. The user can quickly get more data about the nodes at that location by moving the mouse over the node (or tapping it with a finger on a smart phone). The home page also has alerts that are generated by the system as well as user-configurable alerts and a section where the user can make notes. If the user chooses to delve into the data, they can view both real-time data (Fig. 5) and/or generate a chart of historical data (Figs. 6 and 7).

Irrigation control using this WSN system is versatile and has multiple modes (Kohanbash et al., 2012a). Users can manually send manual irrigation commands, configure irrigation schedules, configure local set points, and configure irrigation based on grower tools as outlined in Table 2 . Within Sensorweb, irrigation groups (or zones) can be configured. Users can assign nodes that share the same irrigation settings to a group so that when irrigation settings are changed all the nodes in the group have their irrigation settings updated; the power of WSN-based distributed irrigation is that each node in the group will still independently control irrigation based on its own sensors. This allows a scalable solution for configuring many irrigation nodes in one step while still having the advantage of precise irrigation control within each node's solenoid. The design of the irrigation

\section{Farm Sensorweb}
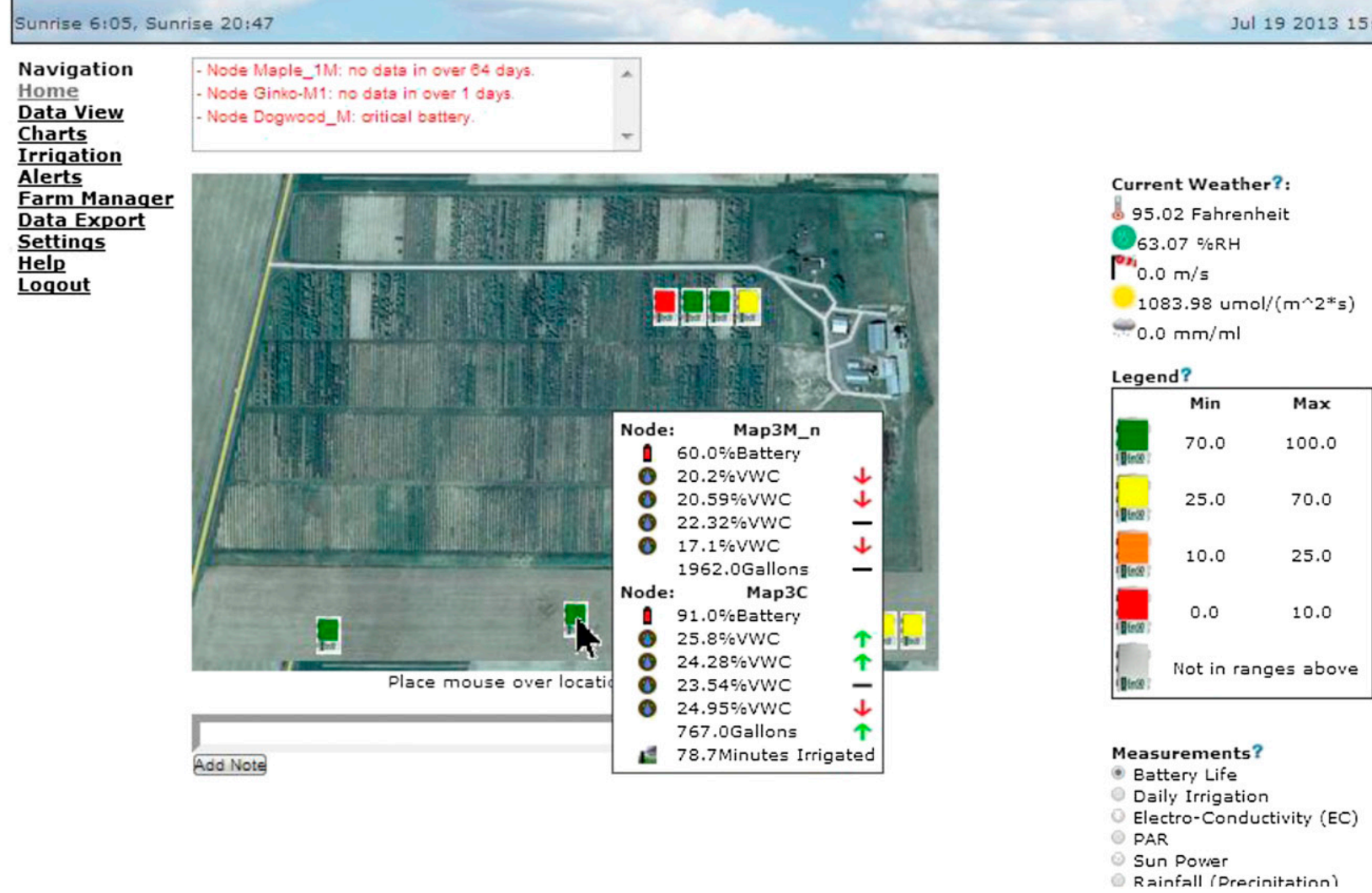

Fig. 4. Screen capture of the Sensorweb home page showing the spatial view and pop up with real-time detail. Arrows next to the data show real-time trends. Alerts in red (upper box) show possible issues with the system as well as user-defined alerts. 


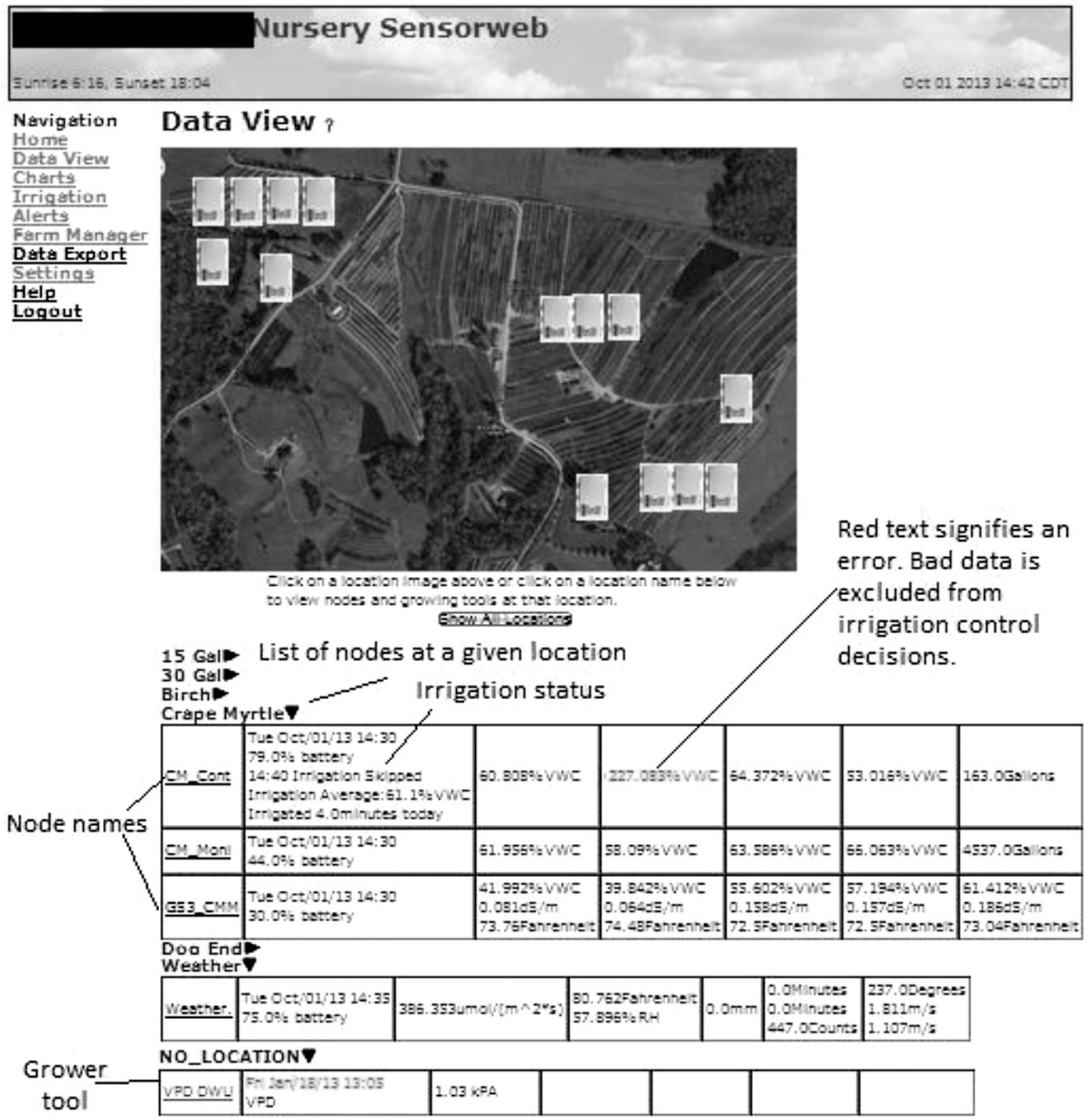

Fig. 5. Screen capture of the Sensorweb data view page (some nodes are hidden to fit the page) showing the recent data from the nodes and grower tools. Clicking on a node name brings up the node configuration page. This example has irrigation control nodes, sensor only nodes, and grower tools.

control page has challenges similar to those of the home page in that each farm has very different irrigation requirements and Sensorweb needs to be able to handle all these needs seamlessly. For this flexibility, irrigation events can be configured for durations ranging from $\mathrm{l}$ s to $\mathrm{l} \mathrm{d}$. The day is broken up into 288 sections of 5 min each that can be scheduled from within the irrigation tool (Fig. 8). If irrigation is enabled within that $5 \mathrm{~min}$ block, a pulse cycle is executed. The pulse cycle is customizable: the user can choose to have irrigation turned on for any portion of the $5 \mathrm{~min}$, with a resolution of $1 \mathrm{~s}$. For example, irrigation can be switched on and off based on some "micropulse" cycle that the user defines (Fig. 9). This feature of being able to configure pulse types has proven to be very valuable. Not only does varying pulse types allow for finer control, it also allows the system to leave time between irrigation pulses to let water percolate through the soil or substrate so that the sensors can react in a more timely fashion to irrigation events. This helps to avoid over irrigation. This is especially true when microsprinklers are used with soilless substrates (Belayneh et al., 2013). Each irrigation method 
Sensorweb

Sunrise 5:54, Sunset 20:31

Jul 192013 16:10 EDT

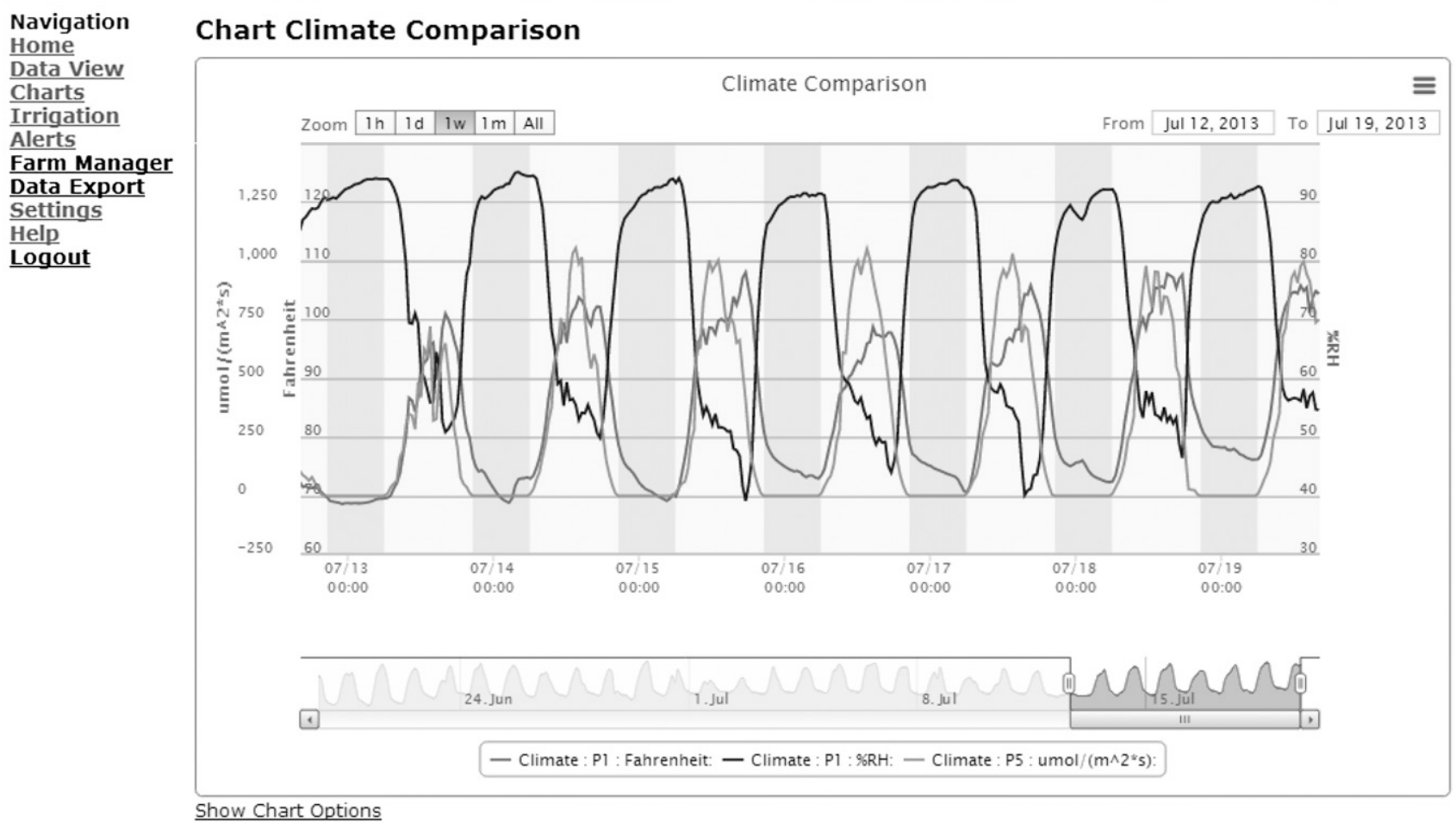

Fig. 6. Sample user configured dynamic chart with node data. Users can zoom in on the data in several ways including selecting the data with the mouse (or finger), the slider bar, or preselected time scales). Yellow (light) and gray (dark) vertical bands signify day and night regions within the chart. Charts are generated using the Highstock library (Highsoft, Vik, Norway).

has a different control methodology. Having the node control irrigation in "local mode" is useful since if the connection to the base station is lost (poor/no radio connection, loss of power, etc.), the node will continue to manage irrigation without interruption. This is very important for system reliability (Majsztrik et al., 2013a); even if other nodes or the base station have a fault, nodes continue to operate independently. In the case of global control where the base station is the control point and makes the irrigation decision the node will revert to using just the local schedule stored on the node if the base station fails. This dual control methodology provides a robust approach for the irrigation system so that nodes can continue irrigation if telemetry is lost.

The database is an SQLite3 filebased database. It is responsible for storing all the settings and data for the WSN system. One of the reasons for choosing SQLite3 as the database is that it is file-based so writes are slower but reads are faster than a serverbased database system. Within the WSN system, there are relatively few inserts into the database, compared with the size of the search queries when the user is accessing the system (particularly when generating plots). Each node is assigned a unique ID in the database. All data are associated with both that unique ID and the hard-coded serial number of the node that the data came from.

The node communication software and the grower tool system need to run continuously in the background as opposed to the user interface which is triggered by user activity, as they access the interface from a web browser. The node communication software uses Perl (2013). Perl is widely supported and has excellent database support. Another big advantage of using a scripting language such as Perl is its built-in parsing functions that make working with data, disassembling incoming data packets, validating the data, and assembling new packets much easier. Grower tools provide advanced features to the user and must be run in the background at predefined schedules. Some examples of grower tools include computing averages, vapor pressure deficit, dew point, evapotranspiration, water savings, and plant models. The grower tool also monitors the current data for sending out user-defined alerts via email or SMS text messaging as necessary. The grower tool software uses Rake and Ruby (Ruby, 1995; Ruby on Rails [RoR], 2003). Rake is bundled with RoR, which is used for the user interface. Although Ruby/Rake might not be the first choice for many scripting languages, it has very powerful and easy to use database tools. It is also tightly integrated with the RoR architecture allowing for the use of functions within RoR in the rake script. In particular, there is a lot of code in the user interface for converting data to 


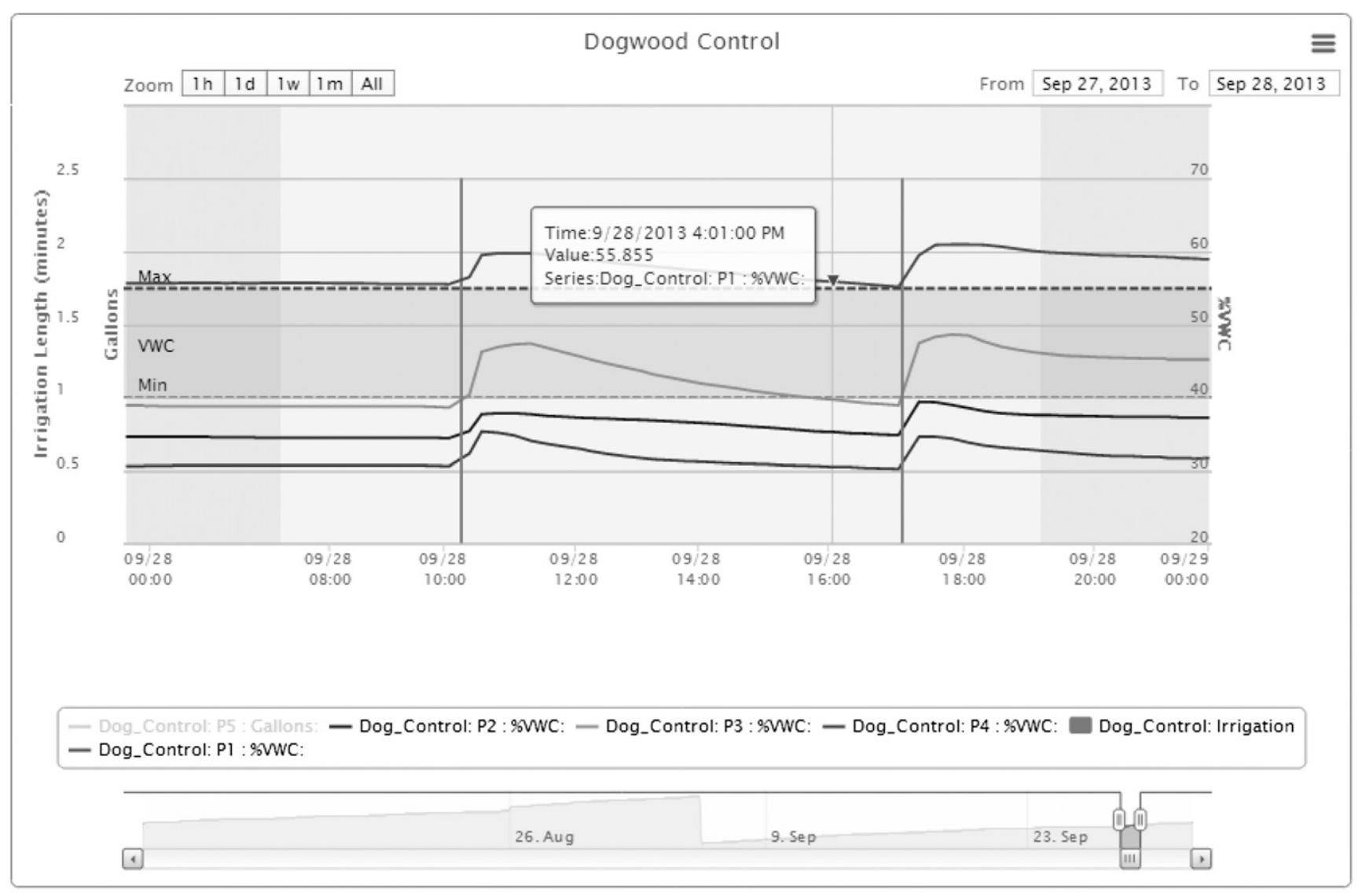

Fig. 7. Sample user configured dynamic chart with node data. Placing the mouse over a data point will show the actual values at that point. The thin vertical lines represent irrigation applied and you can see corresponding jumps in the horizontal soil moisture lines. The dark horizontal band in the middle of the plot is a user-defined region of interest.

Table 2. Various irrigation modes and the benefits of using a wireless sensor network (WSN). The control point is where the irrigation decision takes place.

\begin{tabular}{|c|c|c|c|}
\hline Irrigation mode & Control point & Description & The WSN advantage \\
\hline Manual commands & User & $\begin{array}{l}\text { Allows the grower to send manual } \\
\text { irrigation commands to the nodes }\end{array}$ & $\begin{array}{l}\text { Irrigation can be determined remotely } \\
\text { based on sensor data and sent to the } \\
\text { nodes. Irrigation nodes can also easily } \\
\text { be reconfigured and/or moved based } \\
\text { on the current crop }\end{array}$ \\
\hline Schedule based & Node & $\begin{array}{l}\text { Irrigation is based on a predefined } \\
\text { schedule that is stored in the nodes }\end{array}$ & $\begin{array}{l}\text { Same as manual commands but } \\
\text { a schedule controls irrigation so there } \\
\text { is less direct user involvement }\end{array}$ \\
\hline $\begin{array}{l}\text { Local "set point" } \\
\text { control }\end{array}$ & Node & $\begin{array}{l}\text { Uses the schedule but also looks at the } \\
\text { current soil moisture to determine if } \\
\text { irrigation is needed at that nodes } \\
\text { location }\end{array}$ & $\begin{array}{l}\text { Irrigation is determined at each location } \\
\text { for precise irrigation control }\end{array}$ \\
\hline Global control & Base station & $\begin{array}{l}\text { This is used to control irrigation based on } \\
\text { values external to the node. Examples } \\
\text { of global control can be a sensor on } \\
\text { a different node, a computed value } \\
\text { from a growing tool, or from a plant } \\
\text { science model }\end{array}$ & $\begin{array}{l}\text { This entire method of control is } \\
\text { completely data driven and allows for } \\
\text { feed-forward predictive control }\end{array}$ \\
\hline Pulse types & & $\begin{array}{l}\text { This is a submode for the modes listed } \\
\text { above. With this option, irrigation can } \\
\text { be issued in pulses to allow sensors time } \\
\text { to react, increase precision, or allow } \\
\text { irrigation lines time to recharge }\end{array}$ & $\begin{array}{l}\text { The ability to do this on each node allows } \\
\text { for localized irrigation control. This } \\
\text { can further improve the irrigation } \\
\text { precision by using commanding } \\
\text { micropulses of irrigation as needed }\end{array}$ \\
\hline
\end{tabular}




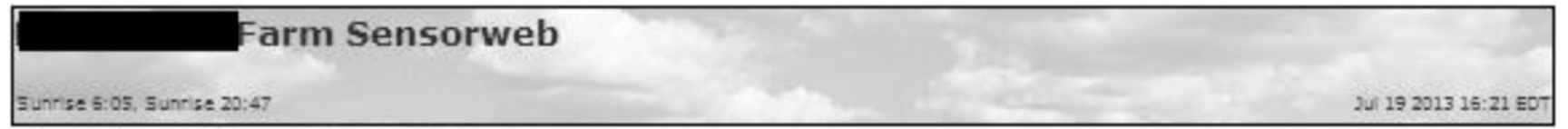

\begin{tabular}{l} 
Narvigation \\
Home \\
Data View \\
\hline Charts \\
Irrigation \\
Alerts \\
Farm Manoger \\
\hline Data Export \\
Settings \\
Help \\
Loqout
\end{tabular}

Irrigation Scheduler

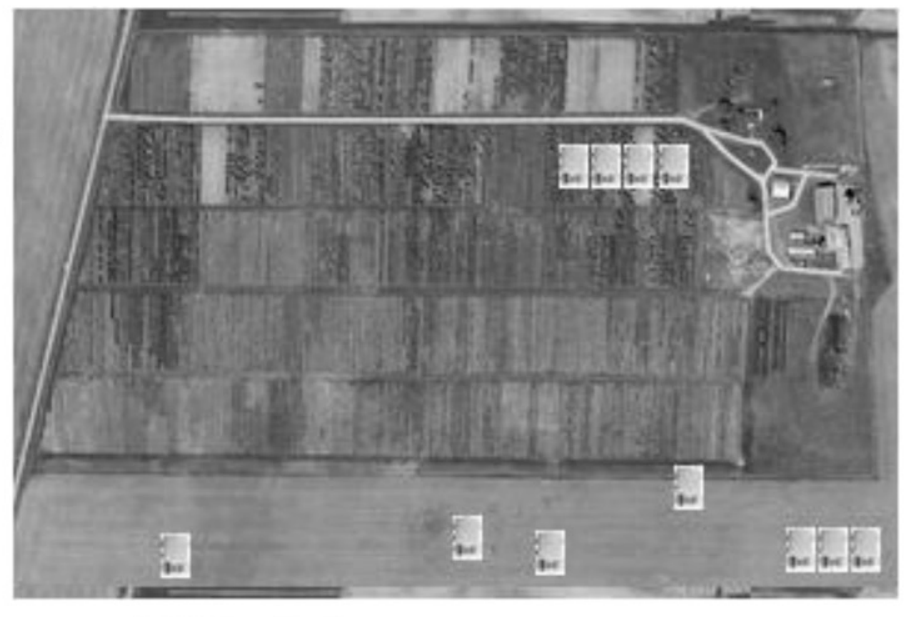

Seisct Node Masie

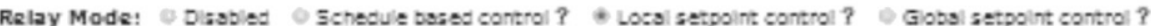

Selsct which molsture sensor ports to uae for control (aelected sensors muat be the same type and will be sversged together):

x Port 10 Fort 20 Port 3 का Port 40 Port 5

Low Setpoint (0-552: Whe): 25

Pulse Type: Fuse 1 $\square$ Edrt pulse bypes here

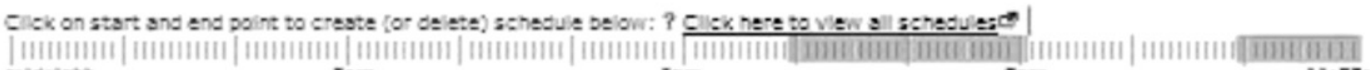

midnight $\quad 3 \mathrm{sm} \quad 6 \mathrm{sm} \quad$ Som $11: 55 \mathrm{sm}$

noon

Gosa Conves Maximum imgationtime per dayla s noura

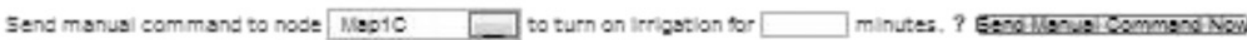

Cnota: It may taka a faw minutes for tha command to taka afoct?

Fig. 8. Screen capture of the Sensorweb irrigation manager page. Blue (i.e., shaded) regions in the schedule are those selected for irrigation.

the proper units and validating data that can be reused within the grower tool scripts.

The grower tool system is designed to be modular so that it is easy to add new grower tools into the system (Kohanbash et al., 2012b). Adding a new grower tool to the system is a two-step process. The first step is to add a line into the database that specifies what the name of the grower tool is, what units the inputs and outputs of the grower tool should be, and where the grower tool script file is. The second step is adding the grower tool script file. When it is time for a grower tool to run (Fig. 10), a standard extensible markup language
(XML) input data file (Fig. 11) is created based on the configuration line in the database and the settings the user selected when instantiating the grower tool. The grower tool script file then reads the input file, runs the grower tool-specific code, and then needs to generate an output XML file (Fig. 12). The output XML file is then read and the data are saved into the database. Once it is in the database, the users can view the data, control irrigation with it, or generate alerts.

\section{Conclusions}

This WSN system has been in use for several years at over a dozen commercial and research sites throughout the United States and has demonstrated its reliability by operating continuously at those sites and accurately controlling irrigation. Using this system has led to financial savings/gains, labor savings, reduced crop growth time, reduced chemical application, and reduced disease/pests for growers that use this system. For a complete discussion of the benefits, see Lichtenberg et al. (2013) and Majsztrik et al. (2013b). The system is continuously evolving based on user feedback and is gaining new features to make WSNs even more valuable and make the data even more actionable. 
Navigation

Home

Data View

Charts

Irrigation

Alerts

Farm Manager

Data Export

Settings

Help

Logout

\section{Relay Pulse Configuration Tool}

This tool allows for the creation of various pulse types that can be used for irrigation. You can configure how long a single pulse should be in seconds. If you want multiple pulses to occur within the $\mathbf{5}$ minute control block you can also specify a time between pulses (in seconds) and the number of desired pulses.

The maximum amount of time a pulse sequence can be defined for is $\mathbf{3 0 0}$ seconds ( 5 minutes) since the irrigation scheduler will run this pulse sequence every five minuted when irrigation is active.

\begin{tabular}{|l|l|l|l|l|l|}
\hline \multicolumn{1}{|c|}{ Name } & Pulse Duration(s) & Time Between Pulses(s) & Number of Pulse Cycles & Total Time of Event \\
\hline Standard & 300 & 0 & 1 & 300 \\
\hline - & MicroPulse & 20 & 20 & 5 & 200 \\
\hline & Pulse 1 & 240 & 60 & 1 & 300 \\
\hline & UNKNOWN TYPE & 280 & 20 & 1 & 300 \\
\hline
\end{tabular}

Name: MicroPulse

Pulse Duration(s) 20

Irrigation On

Irrigation Off -

Time Between Pulses(s.20

Number of Pulses 5

Update current relay pulse type Save as new relay pulse type Delete current relay pulse type

Fig. 9. Pulse type configuration page. This pulse type is showing a micropulse setup, where irrigation is turned on for $20 \mathrm{~s}$, turned off for $20 \mathrm{~s}$, and the cycle is repeated five times for a total irrigation length of $200 \mathrm{~s}$.

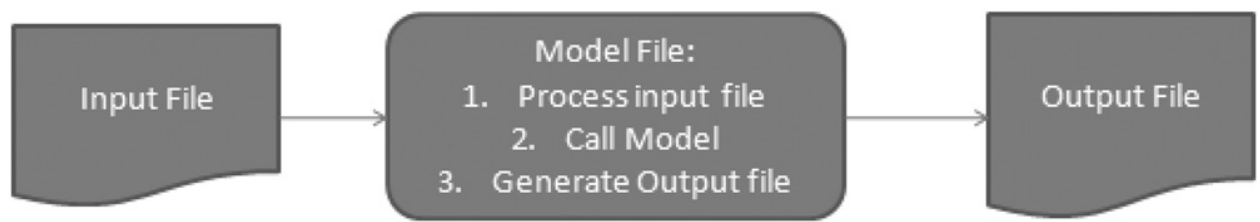

Fig. 10. Grower tool process flow. Showing input file, processing, and output file for running any model using a standard interface.

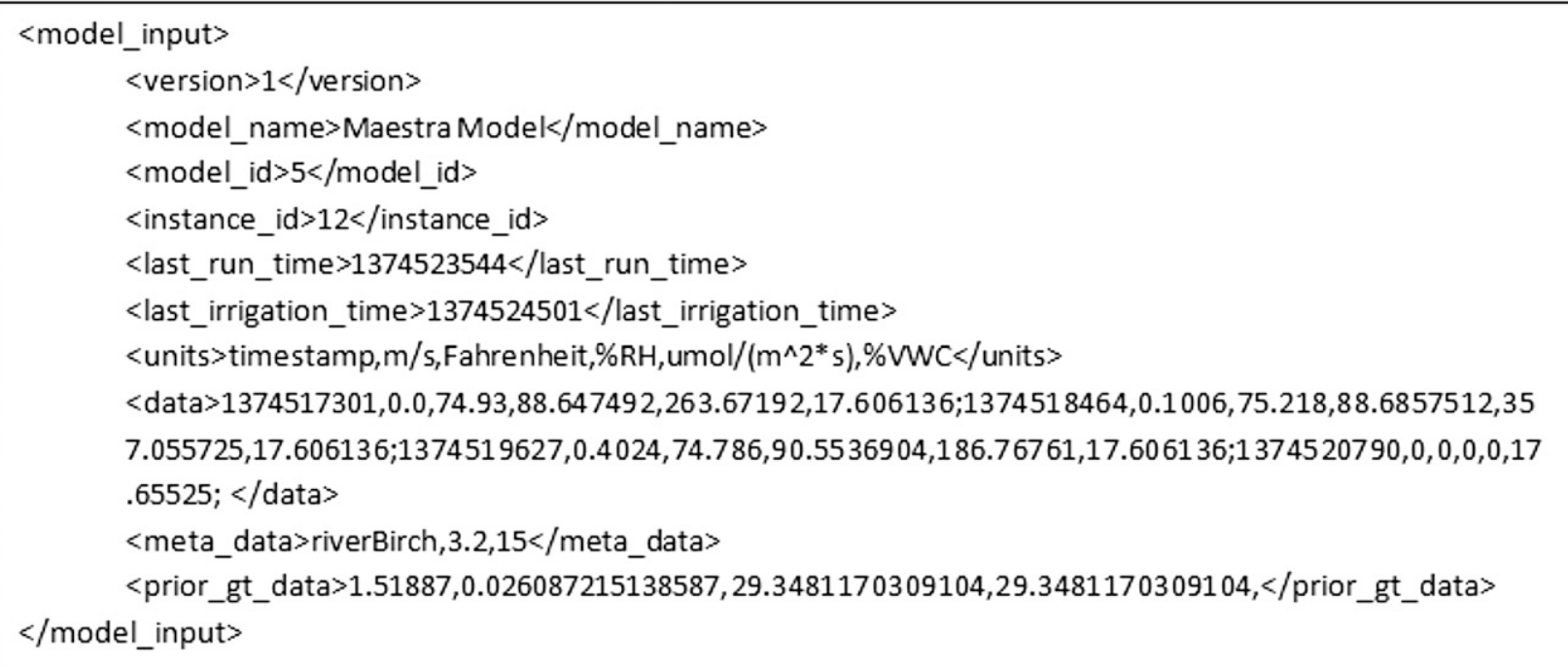

Fig. 11. Sample input file for a grower tool running a plant water use model in a commercial nursery. 


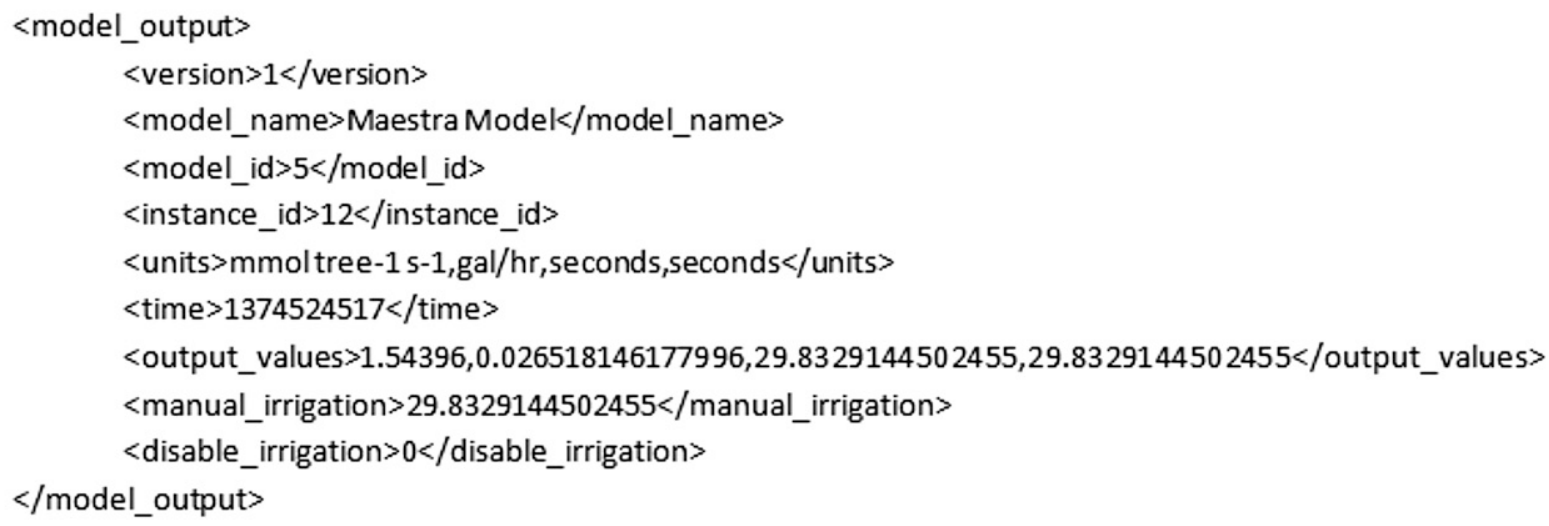

Fig. 12. Sample output file for a grower tool running a plant water use model in a commercial nursery. In this case, the model is commanding $29.8 \mathrm{~s}$ of irrigation that the node will automatically deliver to the block of trees.

\section{Literature cited}

Angelopoulos, C.M., S. Nikoletseas, and G.C. Theofanopoulos. 2011. A smart system for garden watering using wireless sensor networks. Proc. 9th Assn. Computing Machinery Intl. Symp. Mobility Management and Wireless Access. p. 167-170.

Bauerle, T.L., W.L. Bauerle, M. Goebel, and D.M. Barnard. 2013. Root system distribution influences substrate moisture measurements in containerized ornamental tree species. Hort Technology 23: 754-759.

Belayneh, B.E., J.D. Lea-Cox, and E. Lichtenberg. 2013. Costs and benefits of implementing sensor-controlled irrigation in a commercial pot-in-pot container nursery. HortTechnology 23:760-769.

Chappell, M., S.K. Dove, M.W. van Iersel, P.A. Thomas, and J. Ruter. 2013. Implementation of wireless sensor networks for irrigation control in three container nurseries. Hort Technology 23:747-753.

Coates, R.W., M.J. Delwiche, A. Broad, M. Holler, R. Evans, L. Oki, and L. Dodge. 2012. Wireless sensor network for precision irrigation control in horticultural crops. Proc. Intl. Conf. Agr. Eng., Valencia, Spain, 8-12 July 2012.

Kantor, G.A., D. Kohanbash, and T. Martin. 2012. Next-generation monitoring and control hardware development. Proc. Irr. Assn., Orlando, FL, 4-5 Nov. 2012.

Kohanbash, D., A. Valada, and G.A. Kantor. 2012a. Irrigation control methods for wireless sensor networks. Proc. Amer. Soc. Agr. Biol. Eng. Annu. Mtg. Paper No. 121337112.

Kohanbash, D., A. Valada, and G.A. Kantor. 2012b. Base station design and architecture for wireless sensor networks, Proc. Intl. Conf. Agr. Eng., Valencia, Spain, 8-12 July 2012.

Lea-Cox, J.D. 2012. Using wireless sensor networks for precision irrigation scheduling, p. 233-258. In: M. Kumar (ed.). Problems, perspectives and challenges of agricultural water management. InTech Press, Rijeka, Croatia.

Lichtenberg, E., J. Majsztrik, and M. Saavoss. 2013. Profitability of sensor- based irrigation in greenhouse and nursery crops. HortTechnology 23:770-774.

Majsztrik, J., E. Lichtenberg, and M. Saavoss. 2013b. Ornamental grower perceptions of wireless irrigation sensor networks: Results from a national survey. HortTechnology 23:775-782.

Majsztrik, J.C., E.W. Price, and D.M. King. 2013a. Environmental benefits of wireless sensor-based irrigation networks: Case-study projections and potential adoption rates. HortTechnology 23: 783-793.

Perl. 2013. The Perl programming language. 28 Oct. 2013. <http://www.perl.org/>.

Ruby. 1995. Ruby: A programmer's best friend. 28 Oct. 2013. <https:// www.ruby-lang.org/en/>.

Ruby on Rails. 2003. Ruby on Rails. 28 Oct. 2013. <http://rubyonrails.org/>.

van Iersel, M.W., M. Chappell, and J.D. Lea-Cox. 2013. Sensors for improved efficiency of irrigation in greenhouse and nursery production. HortTechnology 23:735-746 